\title{
An in-situ Degradation Behavior Study of MAO Coating on AZ91D Magnesium Alloy in Aqueous Solutions by SECM
}

\author{
Yan Xia ${ }^{1}$, Zejie Zhu ${ }^{1}$, Linrong Chang ${ }^{1}$, Jianqing Zhang ${ }^{1}$, Fahe Cao ${ }^{1, *}$, Junxi Zhang ${ }^{2}$ \\ ${ }^{1}$ Department of Chemistry, Zhejiang University, Hangzhou 310027, China \\ ${ }^{2}$ Shanghai Key Laboratory of Materials Protection and Advanced Materials in Electric Power, \\ Shanghai University of Electric Power, Shanghai 200090, P. R. China \\ *E-mail: nelson_cao@zju.edu.cn
}

doi: $10.20964 / 2017.03 .65$

Received: 20 December 2016 / Accepted: 28 January 2017 / Published: 12 February 2017

\begin{abstract}
The degradation behavior of AZ91D magnesium alloy with micro-arc oxidation (MAO) coating in $\mathrm{NaCl}$ solutions was studied by scanning electrochemical microscopy (SECM). The investigation was carried out at SECM with 10 micron tip using ferrocenemethanol $(\mathrm{FcMeOH})$ as the redox mediator in feedback mode. The variation of localized reactivity of the MAO coating are reflected by tip currents in SECM mapping images, and the generation and evolution of active site/sites on MAO coating are correlated to the concentration of $\mathrm{Cl}^{-}$in the solution and microstructure of the coating. With $\mathrm{Cl}^{-}$ concentration increasing, the rate of generation of active sites is accelerated and meanwhile the reactivity of the active sites is enhanced. For the relatively thick and porous coating, there are lots of independent active sites incubating, generating and evolving on coating with the extension of immersion time. While the coating is relatively thin and smooth, the coexistence of slender and lower current peaks and pieces of active regions appears. The reactivity of the artificial line scratch and surrounding areas varied with the increase of immersion time, indicating that the generation, accumulation and dissolution of corrosion product affect the corrosion behavior of AZ91 alloy.
\end{abstract}

Keywords: AZ91D; MAO coating; SECM; Feedback mode; Degradation behavior

\section{$\underline{\text { FULL TEXT }}$}

(C) 2017 The Authors. Published by ESG (www.electrochemsci.org). This article is an open access article distributed under the terms and conditions of the Creative Commons Attribution license (http://creativecommons.org/licenses/by/4.0/). 